\title{
Emergence et sédimentation de l'identité: la catégorie romand dans des activités d'entretien
}

\author{
Nicolas Pepin \\ Institut d'Etudes françaises et francophones, Université de Bâle \\ Nicolas.Pepin@unibas.ch
}

\section{Introduction}

La Suisse romande n'est ni une entité politique ni une unité administrative. Elle peut se définir comme un espace représentationnel dont le français est l'emblême. En effet, devenu langue territoriale, le français a pris le pas sur les dialectes francoprovençaux et oïliques en se répandant dès le XIIIe siècle pour s'imposer définitivement dans le courant du XIXe siècle et au début du XXe siècle, principalement sous la pression des nouveaux modes industriels (Kristol 2005). Pourtant, du fait que peu d'éléments langagiers permettent de l'isoler comme zone linguistique distincte des provinces françaises de l'Est ou du Sud-Est de la France, la Suisse romande peut être décrite comme une France politiquement suisse ou une Suisse linguistiquement française (Knecht 1979 : 249). Ainsi, la Suisse romande est prise dans une double appartenance qui révèle simultanément une double alterité. Ni Français, ni Alémaniques, les Romands sont des Suisses francophones.

A partir de cette tension fondamentale, de nombreux travaux ont contribué à mieux cerner les modalités de l'identité romande, en thématisant par exemple le statut et la place du français pour les locuteurs, l'influence de l'allemand et du suisse-allemand, les relations avec les autres communautés linguistiques de la Suisse, ainsi que la place de la Suisse romande dans la francophonie. Ces travaux ont été menés essentiellement à l'aide de questionnaires (par exemple Singy 1996, 2004) ou par le biais d'entretiens semi-directifs (par exemple Cichon 1998) à partir desquels se sont développées des études qui ont associé à l'analyse de contenu une analyse des formes langagières et discursives (par exemple de Pietro \& Matthey 1993, Lüdi \& alii 1995, Py \& Oechs-Serra 1997). Dans cette perspective, l'identité est indissociable de la manière dont elle s'exprime dans le discours et des représentations sociales qui y sont mobilisées. D'autre part, des travaux ethnologiques (par exemple Centlivres 1996) se sont intéressés à la fonction des emblêmes pour identifier les membres de la communauté romande et pour se distinguer des autres communautés. Dans ce cadre, l'emblème, par sa lisibilité sociale, est attaché à une caractérisation positive des identités par les acteurs sociaux. En outre, des travaux sociologiques d'orientation ethnométhodologique ont investigué les rapports entre identités collectives et langues nationales (par exemple Widmer 2004). De ce point de vue, le fait d'avoir une langue ne représente pas d'abord une différence linguistique, mais sert avant tout à établir un rapport à soi en tant que collectif publique. Enfin, des travaux en linguistique interactionnelle ont abordé l'identification à la Suisse romande chez des migrants français installés dans cet espace de la Suisse (par exemple Pepin 2007a).

Dans le présent article, j'aborde la question de l'identité en m'intéressant à la manière dont la catégorie /romand/ est activée et gérée dans des entretiens libres réalisés avec des personnes vivant en Suisse romande et ayant le français comme langue première ou comme langue principale ${ }^{1}$. Dans ce cadre, je ne pars pas de catégorisations préétablies, mais j'observe la manière dont les locuteurs se saisissent de cette catégorie pour attester leur identité, en lien avec d'autres catégories et d'autres ressources de l'entretien. J'adopte un point de vue qui décrit les catégories rendues pertinentes par l'interaction en fonction de leur émergence contextuelle. L'analyse, pour traiter des phénomènes qui mêlent conditions immédiates de l'interaction et éléments sédimentés de la construction identitaire, tient compte du statut indexical et réflexif des situations qui fait que toute situation crée en quelque sorte son propre contexte (de Fornel 1999). En outre, pour rendre compte de l'ancrage séquentiel dans lequel les catégories émergent et sont traitées par les locuteurs, l'analyse porte sur des extraits relativement longs. 
Avant de présenter mon approche de l'identité, j'aimerais encore brièvement préciser ma conception de l'entretien d'enquête et de son exploitation analytique. L'entretien sert généralement à recueillir le discours épilinguistique ou métadiscursif d'informateurs. A ce titre, dans une approche inspirée de l'analyse conversationnelle, on a tendance à considérer que l'entretien n'est pas un moyen d'investigation approprié de la réalité sociale ${ }^{2}$. Cependant, l'entretien d'enquête représente un type de situation, partageant un air de famille avec d'autres entretiens, comme l'entretien d'embauche ou l'entretien journalistique par exemple. A ce titre, et bien qu'il présente des spécificités propres et soit porteur d'enjeux partiellement différents ${ }^{3}$, l'entretien d'enquête est descriptible du point de vue de son déroulement et de son organisation interactionnels. Une telle conception est compatible avec une une approche située de l'identité, telle que je la soutiens dans le cadre de cet article. Cette précision n'est pas gratuite. Elle a en effet des conséquences sur l'analyse des entretiens, puisqu'elle permet d'investiguer les procédures linguistiques et interactionelles déployées de manière coordonnée par les interactants, y compris l'intervieweur, pour gérer contextuellement leur(s) identité(s).

\section{Une approche située de l'identité}

La conception de l'identité dans laquelle je m'inscris part du constat qu'il existe des liens étroits entre identité et accomplissement des activités sociales (Williams 2000). Dans cette perspective, l'identité est intimement liée au déroulement de l'interaction et renvoie à l'enchâssement des individus sociaux dans des catégories liées à des traits plus ou moins typiques d'attribution ${ }^{4}$. Une telle approche s'intéresse en particulier aux modes discursifs d'émergence et de gestion de l'enchâssement catégoriel. En d'autre termes, on met l'accent sur la dimension située et dynamique des ressources de catégorisation, dont la pertinence relève de l'orientation des locuteurs. Les identités sont ainsi fonction des activités que les locuteurs accomplissent. Les procédés langagiers et interactifs permettent d'activer les catégories, de les formuler, de les reformuler, de s'y affilier ou au contraire de les rejeter pour attester son identité et contribuer à attester celle des autres. De ce fait, l'identité présente un caractère opératoire ${ }^{5}$, les locuteurs s'orientant vers des catégories différentes en fonction de leurs objectifs, du contexte, de l'activité, des interlocuteurs ou encore du topic. L'enchâssement peut concerner une seule catégorie ou se réaliser dans des constellations impliquant plusieurs catégories.

Ceci dit, le déploiement situé des identités révèle en fait une tension entre émergence et sédimentation, tension qui se gère et se résout provisoirement par un travail d'ajustement catégoriel. Actualisées dans et par l'interaction où elles sont (re)configurées et (re)formulées, les catégorisations sont stabilisée en contexte, de manière plus ou moins consciente ${ }^{6}$. Certes, les conditions liées à la situation d'entretien favorisent une attitude de contrôle et donc d'élaboration consciente de la sphère identitaire, mais pour autant les catégorisations sont avant tout liées à l'accomplissement des activités de l'interaction (raconter, expliquer, évaluer, argumenter, convaincre, décrire, etc.) et la concrétisation de pertinences contextuelles.

\section{Identités, stéréotypes, énonciations}

Du fait qu'ils jouent un rôle important dans les procédés de catégorisation et spécifiquement dans l'ajustement catégoriel des participants, j'accorde dans cet article une attention particulière aux phénomènes stéréotypiques et énonciatifs.

En ce qui concerne les stéréotypes, il est admis qu'ils participent des procédés d'intelligibilité et d'identification mis en oeuvre par les membres d'une société dans leur vie sociale (p.ex. Quasthoff 1989). Dans ce cadre, j'observe que les individus traitent différemment les aspects typiques de la réalité et les aspects non typiques, ceux qui sortent de l'ordinaire et qui nécessitent une explication par exemple. Les stéréotypes permettent ainsi de traiter les apparences normales pour élaborer ce qui s'en détourne (Pepin 2007b). A la suite de Cicourel (1964) et de Mondada (2002), j'observe également que la non-focalisation liée à la forme normale tend à précéder la focalisation sur les aspects non typiques de la réalité ou sur les traits non conventionnels des catégories. Ainsi, les éléments typiques ou conventionnels tendent à être traités prioritairement, les individus sociaux faisant ensuite, si nécessaire, un travail de détermination 
relativement à leurs objectifs, à leurs interlocuteurs, aux cours d'action dans lesquels ils sont engagés et à leur savoir de membres ${ }^{7}$. Dans cette optique, j'aborde les liens entre stéréotypes et identité par l'exploitation que font les locuteurs des formulations stéréotypiques pour modaliser, rejeter ou au contraire prendre en charge les catégories qui leur sont attribuées.

A partir de l'idée que l'identité du sujet passe par la relation qu'il établit avec ce qu'il dit, à savoir à ce que «je» dit et prend en charge, mais aussi à la relation qu'il établit avec ce que son interlocuteur dit et, enfin, avec la situation en tant qu'elle se constitue dans l'interaction, se pose la question de l'hétérogénéité énonciative du «je». Une manière de répondre à cette question touche à ce que Authier-Revuz (p.ex. 1995, 1998) appelle le dédoublement méta-énonciatif de la modalité autonymique. L'énonciation d'un segment verbal s'accomplit en effet sur le mode d'une boucle énonciative se représentant elle-même. En ce sens, les locuteurs emploient divers procédés (incises explicites ou marquages intonatifs par exemple) pour commenter, en quelque sorte, les énoncés qu'ils produisent, relativement à ce qui est dit ou à la manière de le dire. L'hétérogénéité touche donc à la fois au niveau constitutif de l'énonciation et au niveau de sa représentation, dans le rapport du sujet à son dire, en particulier au niveau méta-énonciatif où se joue d'une certaine manière la problématique de la représentabilité de soi par le sujet parlant. A cela s'ajoute le fait que le travail catégoriel des locuteurs peut être auto- ou hétéro-déclenché (p.ex. par une question ou par une hétérocatégorisation énoncée par l'interlocuteur). Les différentes configurations séquentielles du déclenchement participent ainsi, au moins de manière potentielle, à la prise en charge énonciative et aux phénomènes d'hétérogénéité énonciative et méritent d'être prises en compte dans l'analyse.

\section{Procédures d'ajustement autour de la catégorie /romand/}

La notion d'ajustement catégoriel renvoie au travail des locuteurs pour ajuster leur propre catégorisation et celle d'une entité dans laquelle ils s'inscrivent à un degré ou à un autre (Kesselheim 2002, 2003). La notion est particulièrement saillante dans les procédés de catégorisation multiple qui produisent de nombreux phénomènes d'ajustement. Les analyses qui suivent pointent des procédés d'ajustement autour de la catégorie /romand/.

\section{Extrait 1 (durée: $1 \mathrm{~min} .50 \mathrm{sec}$ )}

1. RIC donc vous avez été en: allemagne \ça signifie que vous êtes e: vous pratiquez

2. l'allemand/

3. PHI oui $\backslash$ oui=oui $\not \neq$ bon/ alsacien: $\neg$

4. RIC oui

5. PHI e: . scolarité helvétique $\neg$. donc l'allemand $\=$

6. $\quad$ RIC $=$ donc l'alle[mand

7. PHI [on n'y coupe pas

8. RIC on n'y coupe pas en effet oui . oui

9. PHI bon/ je l'ai un peu perdu par rapport à ce que . je pratiquais . par rapport au niveau que

10. j'avais:/ il y a quelques années $\backslash$. c'était bien supérieur à ce que c'est mainte[nant/

11. RIC [mhmh

12. PHI faute d'un emploi régulier $\$. actuellement puisque je suis plutôt orienté vers la partie

13. francophone du pays $\backslash$ alors qu'avant:

14. 
17. RIC $=$ de la suissel

18.

19. PHI avec beaucoup de colloques en allemagne

$20 . \quad$.

21. RIC oui

22. PHI alors évidemment j'ai un peu perdu . plutôt orienté sur la partie francophone

23. .

24. RIC oui

25. PHI avec quand même tous les séminaires de formation en allemand donc en principe: oui

26. $\quad$ e. jusqu'à y a pas/ . si longtemps $\neg$ quatre cinq ans $\neg$ j'aurais dit bilingue/ et

27. actuellement je dis très $<$ bonne, bonnes $><$ connaissance, connaissances $>$ d'allemand $\backslash$

28.

29. RIC XX c'est déjà bien parce que: y a quand même passablement de de romands qui

30. peuvent pas: . se targuer de plus que d'une pratique e .

31. PHI oui $=$

32. RIC $=$ scolaire/ e

33. PHI ben:/ . nécessité fait loill

34. RIC oui $=$ oui $=[$ oui

35. PHI [si on en a pas? besoin/ on: enfin? $\mathrm{X}$ ça se perd certainement très vite\}

L'extrait 1 s'inscrit dans un développement de l'activité initiale de l'entretien, où il est demandé à PHI de se présenter. Il s'ouvre sur une question de RIC, qui s'appuie sur une donnée biographique introduite par PHI (hors extrait), et lie la pratique de l'allemand à un séjour de plusieurs années de PHI en Allemagne (lignes 1-2). Ce liage déclenche une séquence explicative dans laquelle PHI active une double autocatégorisation. Une première catégorie est condensée dans le lexème «alsacien» (3), alors qu'une seconde est condensée dans le syntagme «scolarité helvétique» (5). Les deux catégories sont enchaînées par un phénomène intonatif de liste qui aboutit dans le segment «donc l'allemand» (5). Ce faisant, PHI modifie le liage opéré par RIC avec la pratique de l'allemand, ceci en substituant au trait catégoriel séjour en Allemagne deux autres traits qu'il apparie dans une structure syntaxique cumulative du type $x+y=z$. L'usage d'une telle structure met en évidence le caractère banal et conventionnel de la catégorisation, ce que le format intonatif (intonation continue après «alsacien» et «scolarité helvétique», mais descendante après «l'allemand»), les micro-pauses qui séparent ces trois segments ${ }^{8}$ et la formulation évaluative «on n'y coupe pas» (7) contribuent, à mon sens, à renforcer. La procédure mise en place par PHI lui permet ainsi de s'orienter vers l'accord («oui $\backslash$ oui=ouil», ligne 3 ) tout en sélectionnant les éléments pertinents de la formulation catégorielle.

RIC s'inscrit alors dans la ligne explicative de PHI, en répétant ses paroles $(6,8)$ et en acquiesçant (double «oui», ligne 8), ce que PHI interprète comme une relance, puisqu'il développe une séquence explicative extensive, dans laquelle la formulation des lignes 3 et 5 sera d'ailleurs modalisée. Dans cette séquence, PHI exploite le matériel topical introduit par RIC à la ligne 1 en utilisant les termes «emploi» (12) et 
«connaissance(s)» (27) et en orientant son argumentation vers la sphère de la perte («je l'ai un peu perdu», 9). D'une part, PHI incorpore cette sphère à une temporalité pointant un avant et un «maintenant» (10); d'autre part, il l'associe à une réorientation professionnelle vers la «partie francophone du pays» (12 et suivantes, 22). Mais surtout il traite la question initiale de RIC (qui portait uniquement sur la pratique de l'allemand) en liant pratiques et connaissances et en s'orientant vers la maîtrise de l'allemand (lignes 9-10: «bon je l'ai un peu perdu par rapport à ce que je pratiquais par rapport au niveau que j'avais c'était bien supérieur»; lignes 26-27: «j'aurais dit bilingue actuellement je dis très bonne(s) connaissance(s) d'allemand»). Ce faisant, PHI passe du traitement d'une activité sociale (pratiquer l'allemand ${ }^{9}$ ) au traitement d'une caractéristique personnelle (être bilingue, avoir des connaissances). Ce développement conduit à une première formulation conclusive «donc en principe oui» (25), qui fait écho à la réponse donnée à la ligne 5, mais qui est modalisée par «en principe». Cette formulation est elle-même réinterprétée en fonction de ce qui a été traité à partir de la ligne 9 autour de la sphère professionnelle et aboutit à une auto-catégorisation qui associe être bilingue au passé («jusqu'à y a pas si longtemps quatre cinq ans j'aurais dit bilingue», ligne 26) et avoir de très bonnes connaissances d'allemand au présent (27).

RIC réagit par une évaluation («c'est déjà bien») suivie d'un segment qui vient soutenir l'évaluation. Ce segment (lignes 29-32), par ce procédé de liage disjonctif qui lie /romand/ à un trait catégoriel conventionnellement disjonctif (la pratique/maîtrise de l'allemand est formulée comme étant un trait conventionnellement atypique de la catégorie /romand/) de cette catégorie, tend à catégoriser PHI comme étant un Romand atypique. Bien que le liage catégoriel soit effectué après une hésitation, un silence et un continuateur de PHI, et bien que «scolaire» vienne saturer «pratique» dans une intonation montante qui paraît manifester le caractère hésitant de la catégorisation ${ }^{10}$, il y a dans cette brève séquence, qui présente aussi une dimension laudative de compliment, une menace potentielle pour PHI relativement à l'appariement catégoriel effectué entre lui et l'activité pratiquer régulièrement et maîtriser l'allemand qu'il s'est attribuée. PHI réagit alors en réajustant son propos pour investir pleinement la catégorie /romand/. Cette opération s'effectue en deux temps. D'abord, PHI produit une formule proverbiale, dont la valeur explicative générale et la forme l'assimilent à une règle (33). Puis, il élabore un segment explicatif subordonné dont la mise en mots se révèle nettement plus hésitante: «si on en a pas besoin on enfin ça se perd certainement très vite» (35). Ce faisant, PHI confirme son inscription dans la catégorie /romand/ tout en réajustant le lien entre cette catégorie et la pratique/maîtrise de l'allemand. Ainsi, à partir de la formule figée «nécessité fait loi» (33), PHI réinvestit la catégorie /romand/ comme membre à part entière, tout en lui associant un trait nouveau et non conventionnel, à savoir la pratique de l'allemand, associé à la biographie de PHI.

\section{Extrait 2 (durée: $1 \mathrm{~min} .26 \mathrm{sec}$.)}

1. RIC tu te sens plutôt suisse romande toi/.. ou suisse/ e

2. SYL e:: j-j-oui . je me sens plutôt suisse romande $\backslash$ je me sens pas française $\backslash$ [et puis\&

3. RIC [ouais

4. SYL \&suisse/ e ... un peu mais non plus pas vraiment . donc ça serait plutôt suisse romande

5. je pense

6. RIC tu dis pas vraiment suisse . à cause de quoi/ ... à cause du fait que c'est pas la même

7. langue qu'en suisse allemande par exemple/ [ici

8. SYL

[e ça aussi $\backslash$. c'est pas la même langue

9.

10.

11. c'est peut-être pas non plus vraiment la même e la même mentalité . et pis j'ai pas

vraiment l'impression de: ... m- m- même je $\neq$ ah non $j e=j e=j e$ suis suisse mais c'est pas un: sentiment très fort chez moi: 
12. RIC ouais

13. SYL c'est peut-être encore pire je me sens neuchâteloise à ce moment-là

14. RIC ah/tu te sentirais plus neuchâteloise que suisse/

15. SYL ouais certainement puisque c'est que je vis=c'est là [que: ... ouais $(2 \mathrm{~s}) \downarrow$ mais c'est\&

16. RIC

[ouais

17. SYL \&pas forcément très bien à dire parce que je ferais mieux (de) me dire suisse et tout ça

18.

20.

21.

(...)

28. RIC

29.

31.

SYL

33.

34.

35. SYL =et pis ça je n'ai pas=je n'ai pas du tout e $\mathrm{j}=$ =je n'ai pas envie de m'assimiler à ça parce 36. que parce que voilà

38.

40 . SYL mhmh ouais

41. SYL =la suisse française

42. (rires)

43. SYL en=en espagne je disais fpuisque: je disais $\neq$ ah/ tu parles français/ alors tu es 44.

47. RIC

48.

49. SYL

50 .

51. française/ et je disais non $\backslash$ je suis $=$ je suis suisse $\backslash$ mais ils étaient $\neq$ ah:/ on parle français en suisse/ alors je disais bon $\backslash$ je suis de la suisse française $\backslash$ et puis=et puis ça m'allait bien aussi ouais .. tu revendiques/ un peu ça e je suis romande en fait quand même/ quand tu peux

ouais parce que c'est vrai que je suis pas française $\backslash$ et pis pour=pis si les gens ne savent pas en fait que tu parles que=que tu parles qu'on parle aussi français en suisse pourquoi ne pas: le dire/ y a pas que je n'ai pas envie d'être suisse allemande non plus/ 
L'enjeu central de l'extrait 2 est l'enchâssement adéquat de SYL relativement à différentes catégories qui sont mobilisées par les interlocuteurs. Certaines catégories ont déjà été activées préalablement dans l'entretien (/suisse/, /romand/, /français/); d'autres émergent dans ce passage (/neuchâtelois/ et /suisse allemand/). J'aimerais ici me concentrer sur deux formulations qui émergent de la parole de SYL: «neuchâteloise» (13) et «suisse française» (41). En effet, ces deux formulations actualisent des enjeux révélés par le traitement des catégories d'appartenance, traitement qui conduit à substituer l'instance de catégorisation Suisse romande par Suisse française.

La question initiale de RIC (1) sélectionne deux catégories potentiellement pertinentes pour identifier SYL: /suisse/ et /suisse romande/. Ce faisant, RIC active un programme de dire en se sentir $x$ qui sera recyclé plusieurs fois avant d'être abandonné et remplacé par un programme en (ne pas) être $x^{11}$ qui est fonctionnel à la fin de l'extrait. La réponse de SYL s'oriente vers une affiliation à la catégorie /romand/. Or, cette affiliation s'organise discursivement de manière complexe. D'abord, SYL hésite («e:: j- j-», ligne 2); puis, elle reprend le matériel lexical de la question de RIC, en ne modifiant que les pronoms («je me sens plutôt suisse romande», 2), mais en mobilisant une catégorie absente de la question. SYL active en effet immédiatement la catégorie /français/ pour la rejeter de manière nette et tranchée («je me sens pas française», 2). Ensuite s'opère un travail d'ajustement par rapport à /suisse/, travail dans lequel SYL procède à une série de modalisations qui concernent son enchâssement dans /suisse romande/ («un peu», «pas vraiment», emploi du conditionnel, «je pense»). RIC (ligne 6 et suivante) thématise alors «pas vraiment suisse» et initie un appariement catégoriel par rapport à /suisse allemand/. Cette procédure, dans laquelle SYL s'inscrit, active un réseau catégoriel par lequel émergent et se sédimente de manière située, autour de la sphère langagière et de la sphère culturelle («mentalités», ligne 9), des éléments de l'identité de la locutrice, entre autres à partir de marqueurs spatiaux de référence (ici vs. Suisse allemande dans le tour de parole de RIC, en 7; «quand tu arrives en suisse allemande» chez SYL, 18) qui contribuent à l'appariement et au traitement de la catégorie /neuchâtelois/ (13). Cette catégorie résulte du processus de catégorisation en creux mis en oeuvre par la locutrice, qui fait un travail d'ajustement en termes graduels, en rapport par exemple à /suisse/, et contrastifs, par exemple lorsqu'elle exploite la sphère catégorielle /suisse allemand/ activée par RIC (lignes 17 et suivantes). C'est d'ailleurs cette sphère qui est mise à profit par SYL pour expliquer et prendre en charge son enchâssement dans /neuchâtelois/ par le biais de son ancrage local («puisque que c'est que je vis c'est là», 15).

Cette auto-catégorisation de SYL en «neuchâteloise» ne va d'ailleurs pas sans poser problème pour la locutrice, comme le montre l'emploi de segments tels que «c'est pire» (13), «à ce moment-là» (13), «c'est pas forcément très bien à dire parce que je ferais mieux de me dire suisse et tout ça» $(15,17$, prononcé à voix plus basse) qui jalonnent son dire. SYL est ainsi prise dans une tension liée à la gestion discursive de son appartenance, entre d'une part des catégories déjà activées dans le cours l'entretien, qui se révèlent tout à la fois inclusives et exclusives (/français/ et/suisse/), et d'autre part des catégories qui émergent du travail de formulation catégorielle mais ne peuvent être prises en charge telles quelles, sans modalisation (/neuchâtelois/). Néanmoins, le travail d'ajustement tend à résoudre cette tension et à stabiliser une catégorisation de soi qui peut être prise en charge par SYL. Cela s'effectue en deux temps. Premier temps, la catégorisation négative des Français permet la transformation de «suisse romande» en «suisse française» (41). Cette nouvelle formulation intervient après un long développement de SYL (ligne 31 et suivantes) en réaction au liage que RIC a réalisé (29-30) entre la locutrice et la catégorie /français/. Ce liage est d'ailleurs réalisé par RIC en recyclant le lexème «mentalités» que SYL avait précédemment activé pour s'exclure de la catégorie /suisse allemand/ (9). Second temps, à partir de la nouvelle formulation, SYL développe brièvement une sorte de document biographique qui vient authentifier l'usage de la formulation «suisse française» («je suis de la suisse française», 45) et attester son enchâssement dans cette catégorie.

La formulation d'une nouvelle instance de catégorisation et le développement immédiatement subséquent d'un document présenté comme biographique (la formulation «je suis de la suisse française» est présentée 
comme une autocatégorisation hétérodéclenchée en discours rapporté) rendent ainsi possible la prise en charge de sa propre catégorisation (ligne 43 et suivantes) par SYL. Cette prise en charge s'effectue par l'émergence et le traitement d'un domaine de pertinence lié au français en Suisse et raccroché à la biographie de SYL. Elle est confirmée par l'orientation que lui donne RIC en termes de revendication («tu revendiques un peu ça je suis romande en fait quand même quand tu peux», 47), orientation qui est assumée par SYL («ouais parce que c'est vrai que») de manière positive grâce à la pertinentisation ${ }^{12}$ précédemment effectuée: «si les gens ne savent pas qu'on parle aussi français en suisse pourquoi pas le dire» (49-51), «on parle aussi français y a aussi y en a aussi» (52). On note au passage que la procédure de stabilisation passe par l'abandon du programme en se sentir $x$ ouvert par la question initiale de RIC. En effet, bien que RIC emploie à nouveau ce programme dans sa question en 28-30, l'autocatégorisation de SYL s'exprime en termes neutralisés et impersonnels («ce serait», qui préfigure «y en a aussi») dans sa réponse (38-41). Ensuite, après un passage par le programme en se dire $x$, c'est le programme en être $x$, lié au récit du document biographique, qui semble s'imposer. On voit ainsi comment le dispositif catégoriel à l'oeuvre dans cet extrait est éminemment lié à des programmes de dire relativement figés et, de la part de SYL, à une neutralisation de la prise en charge énonciative.

Dans l'extrait 2, une première figure stéréotypique apparaît quand SYL justifie son enchâssement dans la catégorie /neuchâtelois/ en mettant en évidence le lien entre langue et appartenance: «mais c'est vrai que quand tu arrives en suisse allemande tu ne comprends rien tu donc directement tu te sens déjà un peu autrement quoi» (18-19). L'affiliation à la catégorie /neuchâtelois/ s'appuie sur une désaffiliation vis-à-vis de la catégorie /suisse/ qui contient le trait parler une langue qu'on ne comprend pas et qui fait qu'on se sent différent. Peu après, une seconde figure stéréotypique apparaît explicitement dans la parole de SYL («bon ça c'est vraiment un cliché», 32). Dans ce tour, SYL refuse l'orientation vers la catégorie /français/ que RIC a ouverte dans le tour précédent. L'usage du stéréotype permet de gérer la non-affiliation à cette catégorie et de rejeter toute assimilation avec les Français, la distinction se faisant ici au niveau des mentalités. On voit d'ailleurs la puissance explicative du stéréotype: celui-ci permet le réajustement de SYL à la catégorie /romand/ en fournissant un argument d'évidence entre les traits attribués aux Français et SYL: «parce que parce que voilà» (35-36). Ainsi, le caractère surdéterminé du stéréotype concernant les Français, traité explicitement comme stéréotypique, permet une catégorisation acceptable de SYL et débouche sur une nouvelle instance de catégorisation qui est porteuse du trait qui parle français. Cette instance permet à SYL d'affirmer une identité qui spécifie ce trait catégoriel que la base lexicale romandlaissait implicite, tout en exploitant les catégories /français/ et /suisse allemand/ et certains traits typiques qui leur sont liés.

\section{Extrait 3 (durée: $1 \mathrm{~min} .32 \mathrm{sec}$.)}

1. MAR moi ce qui me/ je me sens . plu[s] . là où je me sens le plus française/ c'est

2. quand $=c$ 'est quand j'ai des contacts avec des étrangers $\backslash$... à la suisse $\backslash$

3.

4. RIC mhmh

5. ..

6. MAR je sais pas comment dire/ par exemple [récemment/

7. HEL [si $\backslash$ c'est cl]air $\backslash$

8. MAR récemment/ un monsieur/ que je vois souvent/ .. avec qui j’ai toujours échangé juste

9. $\quad$ un bonjour:/

10. RIC oui

$11 . \quad$.. 
12. MAR bon \remarque/ il est pas étranger à la suisse/vu qu'il est suisse allemand $\backslash$. mais enfin/

13.

14. RIC

15. MAR \&retrouvés $\neg$ à descendre $\neg$ les deux $\neg$ la rue $\neg$.. et puis je lui dis $=$ j'ava- $\neq$ je sais pas/

16.

17.

18.

19.

20.

21.

RIC j'ai pris peut-être plus le temps/ ou: tout d'un coup il s'est mis à me parler . et puis on a découvert que: il parle +il était suisse allemand/ et du coup il s'est mis à me parler allemand $\backslash$... et du coup on est descendus $\backslash$. en parlant allemand $\backslash$. et il était heu-reux $\backslash$ $H$ : mais=mais heureux $\backslash$ mais c'était incroyable $\backslash j$ 'en revenais pas parce que . il se

22. MAR

25 .

ou bien où el-= $=$ mais pas suisse romande $\backslash$

où elle se sent française aussi $\backslash$ mais d'une certaine façon $\backslash$.. quand i- les romands ils

29. développent leurs=leurs clichés $\backslash . \Rightarrow \mathrm{ah} /<\mathrm{la}$,le $>$ suisse allemand $/ \Leftarrow$ (accent alémanique

30.

31. RIC caricatural) . bon c'est $=c^{\prime}$ 'est de l'autre côté du: de la=de [la sarine $\backslash$

32.

33. HEL et là ça/ elle a jamais pu comprendre çal

L'extrait 3 s'inscrit dans une séquence qui porte sur l'intégration de MAR en Suisse. MAR a été contactée comme Française pour participer à l'entretien; HEL, son mari, est originaire de Suisse alémanique ${ }^{13}$. Après s'être rencontrés en France, HEL et MAR ont décidé de s'installer en Suisse romande, où ils vivent depuis une douzaine d'années. L'aspect sur lequel j'aimerais insister dans cet extrait touche à la manière dont MAR traite contextuellement son appartenance en l'ajustant à la catégorie /étranger/.

A la ligne 2, dans la préface au récit qui commence à la ligne 6, la catégorie /étranger/ est d'abord liée «à la suisse», puis, à la ligne 13 , dans le récit à proprement parler, elle est liée «à la suisse romande». Dans ce passage, la catégorie /étranger/ apparie deux identités (suisse allemand et français) qui se coalisent autour de l'usage de la langue: parler allemand devient en effet un élément commun aux deux protagonistes de l'histoire racontée par MAR. D'une part, ce trait transforme la relation de MAR au «monsieur», du fait que celui-ci passe de quelqu'un qu'on salue à quelqu'un avec qui l'on parle; d'autre part, le fait de parler allemand en Suisse romande métamorphose le protagoniste de l'histoire, qui devient «heureux mais mais heureux c'était incroyable» (lignes 18-19). La mobilisation de la catégorie /suisse allemand/ permet ainsi l'exhibition d'un sentiment d'étrangéité («il se sent un peu le suisse allemand ici», 19-20) dont le traitement s'ancre dans la situation particulière de MAR (comme Française) et de HEL (comme Alémanique) en Suisse romande. Ce faisant, MAR prend en charge son identité française en mettant à distance la catégorie /romand/ et en investissant la catégorie /étranger/ dans un parallèle qu'elle 
établit avec /suisse allemand/, la catégorie /étranger/ se voyant attribuée un trait (parler allemand) qui la distingue fondamentalement des Romands (en renvoyant au stéréotype que les Romands ne parlent pas l'allemand, cf. extrait 1). MAR utilise ici une formulation tout à fait révélatrice de la manière dont l'ajustement se réalise relativement à la catégorie /romand/: «on était deux non suisses romands», le segment «non suisses romands» étant marqué au niveau de l'intonation et du débit. L'ajustement auquel procède MAR est d'ailleurs thématisé par HEL (ligne 28 et suivantes), dont le tour fait écho à l'évaluation produite à la ligne 7 à propos de la catégorisation préférentielle de MAR en Française, quand elle se trouve «en contact avec des étrangers» (2). Or, la thématisation de HEL vise justement à expliciter les «clichés» (29) des Romands vis-à-vis de /suisse allemand/.

Ici, la sphère stéréotypique est saturée par un phénomène d'écho (la caricature de la caricature de l'accent, 29-30) et par une procédure de localisation et de cloisonnement («de l'autre côté de la sarine», 30) qui permettent de tracer une séparation et de dessiner un mode relationnel entre Romands et Alémaniques. La stigmatisation de l'accent et le rejet au-delà d'une limite géographique symbolique entrent ainsi en contraste avec le récit fait par MAR et viennent expliciter l'ajustement catégoriel de MAR, dans lequel HEL s'intègre prospectivement (évaluation de la ligne 7) et rétrospectivement (développement thématique des lignes 28-30). Le statut stéréotypique du motif de la frontière semble d'ailleurs confirmé par la production quasi collaborative de RIC, qui répète le segment de HEL avec un bref temps de retard et l'évalue par un «oui» (31) à intonation descendante. RIC s'ajuste ainsi dans une partie du réseau catégoriel élaboré par HEL et MAR.

On note enfin que les interventions de HEL dans le récit de MAR met en évidence des éléments catégoriels et narratifs qui semblent sédimenté ${ }^{14}$, mais dont l'émergence dépend du déroulement de l'interaction. De plus, le processus d'ajustement à l'oeuvre dans cet extrait montre que l'identité n'est pas un produit fini, mais au contraire une élaboration contextuelle d'éléments ajustables, dont la prise en charge par les locuteurs est fonction des activités dans lesquelles ils sont engagés.

\section{Conclusion}

Les procédures de catégorisation participent de l'activation et de la gestion des identités en situation. Elles participent de la (re)configuration des appartenance en fonction des activités en cours, en particulier par un travail d'ajustement catégoriel qui mobilise des éléments stéréotypiques. Ce travail d'ajustement concerne évidemment l'inscription des locuteurs dans telle ou telle catégorie, mais touche également à la saturation des catégories, c'est-à-dire aux attributs qui leur sont contextuellement liés. Ce travail d'ajustement est procédural. Il permet de stabiliser, au moins momentanément, les catégories et de sédimenter les identités produites par l'enchâssement, tranché ou graduel, exclusif ou cumulable, des locuteurs dans ces catégories. Dans ce cadre, la sphère stéréotypique peut être convoquée et fonctionalisée de diverses manières. J'ai montré trois cas.

Dans l'extrait 1 , on a vu à l'intérieur d'un tour fonctionnant comme second élément d'une paire autoévaluation - hétéro-évaluation un procédé d'hétéro-catégorisation à potentialité disjonctive. La disjonction est ici liée à un attribut conventionnellement disjonctif de la catégorie /romand/ (à savoir la maîtrise/pratique de l'allemand) qui est associé par RIC à son interlocuteur. Ce procédé donne lieu à un travail de réinscription de l'interlocuteur dans la catégorie/romand/ et de normalisation de son statut. Cela grâce à une formule proverbiale à valeur de règle générale et à l'élaboration d'une explication subordonnée.

Dans l'extrait 2, le travail d'ajustement est réglé par le traitement explicite de stéréotypes liés à la catégorie /français/, afin de traiter le matériel matériel catégoriel dans une formulation qui transforme l'instance de catégorisation «Suisse romande» en «Suisse française». Cette formulation, que la locutrice peut assumer, permet de neutraliser la disjonction entre être neuchâteloise et être suisse, entre romand et français et entre romand et suisse allemand. Par ce dénouement, la catégorisation peut être prise en charge et revendiquée en articulant différents niveaux d'appartenance et en élaborant une identité complexe où les catégories sont liées entre elles dans la procédure d'ajustement. 
Enfin, dans l'extrait 3, le pointage de la sphère stéréotypique est opéré par HEL pour expliciter l'affiliation de MAR dans la catégorie /français/ par le biais de la catégorie /étranger/. L'affiliation a lieu par un ajustement de /français/ et de /suisse allemand/ vis-à-vis de /romand/ à partir de l'usage commun de la langue allemande. La stigmatisation des traits attribués stéréotypiquement à /suisse allemand/, dans une énonciation hétéro-attribuée à /romand/ par HEL, permet d'élucider l'ajustement catégoriel. La coalition entre MAR et le monsieur suisse allemand qui a été réalisée autour de la langue par MAR correspond ainsi à un renversement des stéréotypes attribués aux Romands dans le comportement de MAR. En explicitant cette coalition selon une représentation sédimentée liée aux catégories /suisse allemand/ et /romand/, HEL s'ajuste lui-même aux catégories mobilisées par sa femme.

En outre, dans les trois extraits, le discours rapporté sous sa forme directe (extrait 2: «on est les meilleurs», 32; «je suis de la suisse française», 45; extrait 3: «ah la/le suisse allemand», 29) et les formulations figées (extrait 1: «nécessité fait loi», 33; extrait 3: «c'est de l'autre côté de la sarine», 30) servent à implémenter un dire extérieur à la situation d'élocution dans le discours du locuteur et à lui donner un caractère authentique qui permet de gérer la représentabilité de son identité et sa prise en charge. A ce titre, ces modes de dire jouent et rejouent des enjeux sociaux qui dépassent la situation en cours et expriment une tendance à la stabilisation contextuelle des identités. En effet, les procédés utilisés par les locuteurs servent à règler des conflits de structuration identitaire qu'ils contribuent concrètement à exhiber et opèrent en rendant fonctionnelles des catégorisations plus ou moins sédimentées, mais dont la manifestation est liée à la situation.

Dans cette perspective, c'est l'interaction qui contribue à faire émerger et à gérer les appartenances des locuteurs à des communautés. En particulier, les locuteurs peuvent s'attribuer ou se voir attribués, à des degrés divers mais toujours en fonction du déroulement interactif (topics, interlocuteurs, situations, ...), différentes identités, dont l'élaboration située rend compte d'enjeux sociaux qui s'incorporent dans les procédés de catégorisation, entendus comme lieux d'inclusion ou d'exclusion et comme lieux d'identification et de différenciation. A chaque fois, la catégorie /romand/ est au coeur de ces enjeux.

\section{Notes}

${ }^{1}$ Une cinquantaine d'entretiens réalisés en français entre 1997 et 2004.

${ }^{2}$ C'est la position de fond de l'analyse conversationnelle.

${ }^{3}$ Cf. par exemple Pepin (2002).

${ }^{4}$ Cf. par exemple Antaki \& Widdicombe (1998), Czyzewski\&alii (1995), Hester \& Housley (2002), Sacks (1972).

${ }^{5}$ Sacks (1992 : II 327).

${ }^{6}$ La dimension réflexive de l'entretien favorise la prise de conscience. Brès (1989: 175) rapporte ainsi que «le récit éclaire le sujet: bien souvent «je ne sais ce que j'ai fait que lorsque je le raconte». Cf. aussi Ricoeur (1990) pour qui l'identité (narrative) est une mise en abîme de soi-même comme un autre.

${ }^{7}$ C'est le cas à des degrés divers dans les extraits analysés dans cet article.

${ }^{8}$ Plus précisément, PHI ne produit la première pause qu'après l'intervention de RIC à la ligne 4 , suite à une forme de continuateur («e:»).

${ }^{9}$ Les termes en italiques sont des reformulations de l'analyste.

${ }^{10}$ Ce que semble confirmer le segment «e» (32).

${ }^{11}$ En fait, les deux programmes se réalisent également sous forme négative dans cet extrait.

12 J'emprunte le terme à Mondada (1999).

${ }^{13}$ Catégories qui ont été thématisées dès le début de l'entretien, /français/ par RIC, /suisse allemand/ par HEL. 
${ }^{14}$ Cf. p.ex. la manière qu'a HEL d'évaluer ou de commenter ce que dit sa femme (ligne 7), la manière qu'il a d'expliquer le sens des propos de MAR (ligne 33), la manière qu'il a de contribuer à sa catégorisation et de s'ajuster à son dire (lignes 26 et suivantes).

\section{Références bibliographiques}

Antaki, Ch. \& Widdicombe, S. (1998). Identity as an achievement and as a tool. In Antaki, Ch. \& Widdicombe, S. (éds), Identities in Talk. London : Sage. 1-14.

Authier-Revuz, J. (1995). Ces mots qui ne vont pas de soi. Boucles réflexives et non-coïncidences du dire. Paris : Larousse.

Authier-Revuz, J. (1998). Enonciation, méta-énonciation, hétérogénéités énonciatives et problématiques du sujet. In Vion, R. (éd.), Les sujets et leurs discours. Enonciation et interaction. Aix-en-Provence : Université de Provence. 63-79.

Brès, J. (1989). Praxis, production de sens/d'identité, récit, Langages, 93, 23-44.

Centlivres, P. (1996). L'identité régionale: langage et pratiques. Approches ethnologiques, Suisse romande et Tessin. In Centlivres P. \& alii (éds), Les Sciences Sociales face à l'Identité Régionale: Cinq Approches, Berne : Haupt. $77-126$.

Cichon, P. (1998). Sprachbewusstsein und Sprachhandeln. Romands im Umgang mit Deutschschweizern. Wien : Braumüller.

Cicourel, A. (1964). Method and measurement in sociology. New York : Free Press.

De Pietro, J.-F. \& Matthey, M. (1993). Entre insécurité et identité linguistiques: le cas de Neuchâtel (Suisse). CILL, 19, 3-4, 121-133.

Fornel, M. de (1999). Indexicalité, dépendance contextuelle et situations. In Fornel, M. de \& Quéré, L. (éds), La logique des situations. Raisons pratiques, 10, 119-128.

Kesselheim, W. (2002). National identity in interaction: the Argentine case. In Hester S. \& Housley W. (éds), Language, Interaction and national identity. Studies in the social organisation of national identity in talk-ininteraction. Ashgate : Hants. 169-193.

Kesselheim, W. (2003). Prozesse der Gruppenkonstitution: die sprachliche Herstellung von Gruppen im aktuellen argentinischen Einwanderungsdiskurs. Université de Bielefeld : Thèse de doctorat non publiée.

Knecht, P. (1979). Le français en Suisse romande: aspects linguistiques et sociolinguistiques. In Valdman, A. (éd.), Le français hors de France. Paris : Champion. 249-258.

Knecht, P. \& Rubattel, Ch. (1984). A propos de la dimension sociolinguistique du français en Suisse romande. Le français moderne, 52, 138-150.

Kristol, A. (2005). Le passage au français : garantie du maintien de la romanité de la Suisse romande. Forum Helveticum, 15, 150-155.

Lüdi, G., Py, B. \& alii (éds, 1995). Changement de langage et langage du changement. Aspects linguistiques de la migration interne en Suisse. Lausanne : L'Age d'Homme.

Mondada, L. (1999). L'accomplissement de l'étrangéité dans et par l'interaction: procédures de catégorisation des locuteurs. Langages, 134, 20-34.

Mondada, L. (2002). La ville n'est pas peuplée d'êtres anonymes: processus de catégorisation et espace urbain. Marges linguistiques, 3, 72-90.

Pepin, N. (2002). L'entretien directif comme lieu de représentation du monde. Mobilisation de ressources biographiques et conversationalisation, Bulletin VALS-ASLA, 76, 117-131.

Pepin, N. (2007a). Identités fragmentées. Eléments pour une grammaire de l'identité. Berne : Peter Lang.

Pepin, N. (2007b). Stéréotypes en interaction. Eléments d'une grammaire de l'identité. In Boyer, H. (éd.), Stéréotypage, stéréotypes. Fonctionnements ordinaires et mise en scène. Tome 2: Identité(s). Paris : L'Harmattan.

Py, B. \& Oechs-Serra, C. (1997). Le crépuscule des lieux communs, ou les stéréotypes entre consensus, certitude et 
doute. Tranel, 27, 29-49.

Quasthoff, U. (1989). Social Prejudice as a Resource of Power: Towards the Functional Ambivalence of Stereotypes. In Wodak, R. (éd.), Language, Power and Ideology. Amsterdam : Benjamins. 181-196.

Ricoeur, P. (1990). Soi-même comme un autre. Paris : Le Seuil.

Sacks, H. (1972). An initial investigation of the usability of conversational data for doing sociology. In Sudnow, D. (éd.), Studies in Social Interaction. New York, London: The Free Press, Collier-MacMillan. 31-74.

Sacks, H. (1992). Lectures on conversation. Oxford, Cambridge : Blackwell.

Singy, P. (1996). L'image du français en Suisse romande. Paris : L'Harmattan.

Singy, P. (éd., 2004). Identités de genre, identités de classe et insécurité linguistique. Berne : Lang.

Widmer, J. (2004). Langues nationales et identités collectives. L'exemple de la Suisse. Paris : L'Harmattan.

Williams, R. (2000). Making identity matter: identity, society and social interaction. London : Sage.

\section{Conventions de transcription}

[ ] Chevauchement (début et fin)

$=\quad$ Enchaînement immédiat

\& Continuation du tour sur une autre ligne

..... Pauses en tiers de seconde

(3s) Pause en secondes

- Troncation

: Allongement

/ Intonation montante

$1 \quad$ Intonation descendante

$\neg \quad$ Intonation progressive

$\neq \quad$ Rupture intonative

gras Emphase

$+\quad$ Accélération débit

- $\quad$ Ralentissement débit

$\uparrow \quad$ Voix plus forte

$\downarrow \quad$ Voix moins forte

$\mathrm{H} \quad$ Rire

X Syllabe incompréhensible

? $\quad$ Doute du transcripteur

$<\mathrm{x}, \mathrm{y}>\quad$ Propositions multiples 